\title{
The solvation of $\mathrm{Cl}^{-}, \mathrm{Br}^{-}$, and $\mathrm{I}^{-}$in acetonitrile clusters: Photoelectron spectroscopy and molecular dynamics simulations
}

\author{
Gil Markovich \\ School of Chemistry, Tel-Aviv University, Tel-Aviv 69978, Israel \\ Lalith Perera and Max L. Berkowitz \\ Department of Chemistry, University of North Carolina, Chapel Hill, North Carolina 27599 \\ Ori Cheshnovsky \\ School of Chemistry, Tel-Aviv University, Tel-Aviv 69978, Israel
}

(Received 1 March 1996; accepted 19 April 1996)

\begin{abstract}
We present the photoelectron spectra of $\mathrm{Cl}^{-}, \mathrm{Br}^{-}$, and $\mathrm{I}^{-}$solvated in acetonitrile clusters $\left(\mathrm{CH}_{3} \mathrm{CN}\right)_{n}$ with $n=1-33,1-40$, and 1-55, respectively, taken with $7.9 \mathrm{eV}$ photon energy. Anion-solvent electrostatic stabilization energies are extracted from the measured vertical electron binding energies. The leveling of stabilization energies beyond $n=10-12$ for the three halides signifies the completion of the first solvation layer. This is different from the behavior of anion-water clusters which probably do not fill the first solvation layer, but rather form surface solvation states. Classical molecular dynamics simulations of halide-acetonitrile clusters reproduce the measured stabilization energies and generate full solvation shells of 11-12, 12, and 12-13 solvent molecules for $\mathrm{Cl}^{-}, \mathrm{Br}^{-}$, and $\mathrm{I}^{-}$, respectively. Ordered shell structures with high stability were found for the clusters of $\mathrm{Cl}^{-}$, $\mathrm{Br}^{-}$, and $\mathrm{I}^{-}$with $n=9,9$, and 12 . This special stability is reflected in the intensity distribution of the clusters in the mass spectra. Larger anion-acetonitrile clusters have the molecules beyond the first solvation layer packed in a small droplet which is attached to the first layer. It is suggested that in general, anions solvated in large clusters of polar solvents, might be located close to their surface.

(c) 1996 American Institute of Physics. [S0021-9606(96)02328-8]
\end{abstract}

\section{INTRODUCTION}

The study of solvated ion clusters provides detailed information on microscopic interactions of ions with their solvent. Better ion-solvent and solvent-solvent interaction potentials may arise from experiments on small solvated-ion clusters. In studies of larger clusters, containing tens of solvent molecules, or more, the evolution of solvation from the confined geometry to the bulk may be inferred.

Photoelectron spectroscopy (PES) is an important tool for studying anion-solvent interactions, both in isolated clusters ${ }^{1-5}$ and thin films of bulk solution. ${ }^{6-8}$ It measures the binding energy of the anionic electron in the cluster, which is directly influenced by the ion-polar solvent interaction potential, distance, and orientation. Due to the vertical nature of the photodetachment, solvent-solvent interactions have hardly any effect on the energetics of the process. ${ }^{9}$ The photodetachment energetics can be calculated by molecular dynamics simulations of ion solvation by switching off the interactions, which are related to the anion, or alternatively by removing the highest occupied molecular orbital electron in $a b$ initio calculations. This feature enables direct comparison of the PES binding energies with theory. Note that this feature is unique to the studies of anion solvation, where the initial state in the photodetachment process is the equilibrium-state solvated anion. There is no equivalent experimental scheme for solvation studies of cations by photodetachment.

In a previous work in our laboratory, Markovich et al. have studied the solvation of halogen anions in water clusters by using PES, ${ }^{5}$ while Perera et al. have used molecular dynamics to simulate this system. ${ }^{10}$ On the basis of comparison of the experimental results with different calculations, we deduced that each of the ions $\mathrm{I}^{-}, \mathrm{Br}^{-}$, and $\mathrm{Cl}^{-}$is attached to the surface of the water clusters. ${ }^{11}$ This behavior may be rationalized in terms of balance between ion-solvent and solvent-solvent interactions. In the case of anion-water clusters, the solvent-solvent hydrogen bonding interaction dominates, and a maximal number of hydrogen bonds is formed, with only partial stabilization of the anion by the solvent molecules.

Perera and $\mathrm{Amar}^{12,13}$ have analyzed the behavior of different neutral solutes in argon clusters in terms of the strength of solute-argon interaction relative to argon-argon interaction. They found that relatively small solute-argon interactions would produce a surface solvation state, while as the solute-argon Lennard-Jones parameter $-\epsilon$ exceeds 1.5 times the $\operatorname{Ar}-\mathrm{Ar} \epsilon$ parameter, the system forms an interior solvation state. Although systems of ions solvated by polar solvents will probably not fall into the same category as these weakly bound van der Waals complexes, the general idea applies: As ion-solvent interaction becomes much stronger than solvent-solvent interaction, the cluster will have a larger probability of forming interior solvation states. This condition is fulfilled in anion- $\mathrm{Ar}_{n}$ clusters, where the anion-induced dipole interaction overcomes the week argonargon interaction. PES experiments by Bowen and co-workers ${ }^{2}$ on $\mathrm{O}^{-} \mathrm{Ar}_{n}$ and by Neumark and co-workers on $\mathrm{I}^{-} \mathrm{Ar}_{n},{ }^{14}$ and on $\mathrm{Br}^{-}\left(\mathrm{CO}_{2}\right)_{n}$ and $\mathrm{I}^{-}\left(\mathrm{CO}_{2}\right)_{n}$ (Ref. 3) have 
shown that these systems produce symmetric solvation shell structures.

In this work we present photoelectron spectra of halides: $\mathrm{Cl}^{-}, \mathrm{Br}^{-}$, and $\mathrm{I}^{-}$, solvated in acetonitrile clusters $-\left(\mathrm{CH}_{3} \mathrm{CN}\right)_{n}$. Acetonitrile is commonly used as an aprotic organic solvent with good electrolyte solvation properties. It was chosen due to the combination of high dipole moment (3.92 D) and lower propensity to form hydrogen bonds. These properties result in favorable ion-solvent interaction (relative to water), possibly leading to full solvation of the anions in the acetonitrile clusters, in contrast with the anionwater clusters' behavior.

We analyze the results using classical molecular dynamics (MD) simulations of halogen anions solvated in acetonitrile clusters. A new potential function has been devised for these simulations, since the existing potentials, used to describe bulk acetonitrile properties, were not able to reproduce the experimental ion-solvent electrostatic interactions properly. Berkowitz and co-workers ${ }^{10}$ have shown in their work on the anion-water clusters, that polarization effects in such systems play a very important role in the energetics and structure of the clusters. We have therefore modified the six site model for acetonitrile by Bohm, McDonald, and Madden ${ }^{15}$ to include polarization.

\section{METHODS}

\section{A. PES experiment}

The experimental setup is described in detail elsewhere. ${ }^{5}$ The clusters are formed in a pulsed supersonic expansion of 1-2 bar of Ar, 40 mbar of $\mathrm{CH}_{3} \mathrm{CN}$, and about 1 mbar of a halogen-containing compound $\left(\mathrm{CCl}_{4}, \mathrm{C}_{6} \mathrm{H}_{5} \mathrm{Br}\right.$, or $\left.\mathrm{C}_{7} \mathrm{H}_{7} \mathrm{I}\right)$. Between 100 and $200 \mathrm{eV}$ electrons are injected into the early stages of the expansion in order to form the charged clusters. The clusters are cooled by further flow in the expansion, pass through a skimmer to a second vacuum chamber, and are mass separated by a reflectron time-of-flight (TOF) mass spectrometer.

Prior to photodetachment the ions are mass selected by an electrostatic mass gate, and an electric field impulse is used to slow the ions down to $\sim 20-100 \mathrm{eV}$ kinetic energy in order to reduce Doppler broadening. About $2 \mathrm{~cm}$ downstream, a vacuum ultraviolet laser pulse intersects the ion beam, and the kinetic energy distribution of the detached electrons is analyzed in a $250 \mathrm{~cm}$ "magnetic bottle" TOF photoelectron spectrometer. ${ }^{16}$

All the spectra are taken with $7.9 \mathrm{eV}$ photons, generated by $\mathrm{F}_{2}$ excimer laser. The spectrometer is calibrated with spectra of the halogen anions $\left(\mathrm{Cl}^{-}, \mathrm{Br}^{-}, \mathrm{I}^{-}\right) .{ }^{17}$

\section{B. MD simulation}

We have tried to use the six-site model of Bohm, McDonald, and Madden ${ }^{15}$ which contains a partial charge and a Lennard-Jones center for each of the atoms in the acetonitrile molecule but does not include polarization terms. This model is able to account for liquid acetonitrile properties, such as neutron scattering results. Del-Mistro and Stace ${ }^{18}$ made use of these potential parameters in simulations of the IR frequency shifts in small acetonitrile clusters as measured by Buck and Ettischer. ${ }^{19}$ Their calculations maintain reasonable agreement with the experiment and reproduce the same structures which were deduced from the experimental work of Schermann and co-workers. ${ }^{20}$ We could not fit our PES experimental results with this model, apparently due to the existence of strong polarization effects in our charged clusters.

In our calculations we have kept the Lennard-Jones parameters of the Bohm potential, yet modified this potential by making the following two changes.

(i) The introduction of a new charge distribution which rests on a more recent $a b$ initio calculation on acetonitrile. ${ }^{21}$ In this work, except for the acetonitrile itself, the association energy of $\mathrm{Cl}^{-}\left(\mathrm{CH}_{3} \mathrm{CN}\right)_{1}$ was calculated. It was in good agreement with the experimental result by Hiraoka, Mizuse, and Yamabe. ${ }^{22}$ The charge distribution from this calculation overestimated the acetonitrile dipole moment $(4.42 \mathrm{D})$. We have uniformly reduced the charges by $10 \%$ to roughly match the experimental value of the gasphase dipole moment of acetonitrile (3.98 D versus the experimental $3.92 \mathrm{D}$ ). This procedure is similar to the construction of the POL model for water, ${ }^{23}$ where the charge distribution was also reduced by a constant factor upon the inclusion of polarization effects in the potential.

(ii) The introduction of polarization effects by attributing polarizability to the anion and to each atomic site.

In our model, anion potentials also include Coulomb, Lennard-Jones, and polarizability terms. The parameters for the anions were taken from halide-water simulations. Some of the Lennard-Jones radii were slightly corrected in order to obtain a better fit to the experiment. The Lennard-Jones parameters of the anions (especially the diameter) constitute the main difference between the different halides, within the model that we use. The results of the simulations and their fit to the experimental values are, therefore, very sensitive to the magnitudes of these parameters.

The simulation method was similar to the one used previously for the halide-water system. ${ }^{10}$ The polarization energy could be calculated both in a self-consistent manner as in the POL model for water ${ }^{23}$ (iterative calculation), or approximately (one-step calculation), by using the electric field generated at a site by the permanent atomic charges of the other molecules (or ion) to calculate the polarization energy at the site, neglecting the correction to this field by the other induced dipoles. We noticed, that the values of the polarization energy calculated in the two methods differed by a few percent. This difference is negligible relative to the total energy of a cluster. Therefore, we have decided to use the economical method, using the approximate values of the electric field.

We did not include a three-body interaction term (solvent-solvent-ion) since, as in the case of the halidewater simulations, its contribution to the total energy of a 
TABLE I. Potential parameters used in the simulations.

\begin{tabular}{|c|c|c|c|c|c|c|c|c|c|c|}
\hline & $\mathrm{H}$ & $\begin{array}{c}\mathrm{C} \\
\text { methyl }\end{array}$ & $\begin{array}{c}\mathrm{C} \\
\text { nitrile }\end{array}$ & $\mathrm{N}$ & $\mathrm{Cl}^{-}$ & $\mathrm{Cl}$ & $\mathrm{Br}^{-}$ & $\mathrm{Br}$ & $\mathrm{I}^{-}$ & I \\
\hline $\mathbf{q}(e)$ & 0.220 & -0.582 & 0.332 & -0.410 & -1 & 0 & -1 & 0 & -1 & 0 \\
\hline$\sigma_{i i}{ }^{a}(\AA)$ & 2.1 & 3.0 & 3.4 & 3.3 & 4.32 & 3.7 & 4.7 & 4.0 & 5.15 & 4.63 \\
\hline $\begin{array}{l}\epsilon_{i i}{ }^{\mathrm{a}} \\
(\mathrm{kcal} / \mathrm{mol})\end{array}$ & 0.02 & 0.1 & 0.1 & 0.1 & 0.1 & 0.1 & 0.1 & 0.1 & 0.1 & 0.1 \\
\hline$\alpha\left(\AA^{3}\right)$ & 0.135 & 0.878 & 0.360 & 0.520 & 3.25 & 2.30 & 4.53 & 3.05 & 6.90 & 5.50 \\
\hline
\end{tabular}

${ }^{\mathrm{a}}$ The transformation from these atomic parameters to $A_{i j}$ and $C_{i j}$, used in Eq. (3) are $\epsilon_{i j}=\sqrt{\epsilon_{i i} \epsilon_{j j}} ; \sigma_{i j}=\left(\sigma_{i i}+\sigma_{j j}\right) / 2 ; A_{i j}=4 \epsilon_{i j} \sigma_{i j}^{12} ; C_{i j}=4 \epsilon_{i j} \sigma_{i j}^{6}$.

cluster is probably small, and the parameters of such a term for acetonitrile were not available to us.

The total potential energy of a cluster is given by

$$
U_{\mathrm{tot}}=U_{\mathrm{el}}+U_{\mathrm{LJ}}+U_{\mathrm{pol}} \text {, }
$$

where

$$
\begin{aligned}
& U_{\mathrm{el}}=\sum_{i>j} \frac{q_{i} q_{j}}{r_{i j}}, \\
& U_{\mathrm{LJ}}=\sum_{i>j}\left(\frac{A_{i j}}{r_{i j}^{12}}-\frac{C_{i j}}{r_{i j}^{6}}\right), \\
& U_{\mathrm{pol}}=-\frac{1}{2} \sum_{i} \alpha_{i} \mathbf{E}_{i}^{2},
\end{aligned}
$$

where the Coulomb field, $\mathbf{E}_{i}$, at each site is given by:

$$
\mathbf{E}_{i}=\sum_{j} \frac{q_{j}}{r_{i j}^{2}} \hat{\mathbf{r}}_{i j} .
$$

The sums are over all the sites, or site pairs of different molecules, in the cluster. $A_{i j}$ and $C_{i j}$ are either homoatomic or heteroatomic Lennard-Jones coefficients, $\mathbf{r}_{\mathbf{i j}}$ are interatomic vectors, $q_{i}$ are the site charges, and $\alpha_{i}$-site polarizabilities. Table I.

The parameters used in the simulations are listed in

In the simulations we calculate the stabilization energy of the anion by the solvent, $E_{\text {stab }}(\mathrm{MD})$, which is the quantity to be compared with experimental results. It is evaluated as the difference between the initial-ionic state and the finalneutral state (at the same nuclear coordinates) which is the result of a vertical electron detachment transition:

$$
E_{\mathrm{stab}}(\mathrm{MD})=\left(U_{\mathrm{el}}^{\mathrm{i}-\mathrm{s}}+U_{\mathrm{LJ}}^{\mathrm{i}-\mathrm{s}}+U_{p}^{\mathrm{ion}}\right)-\left(U_{\mathrm{LJ}}^{\mathrm{n}-\mathrm{s}}+U_{p}^{\mathrm{ntl}}\right),
$$

where the superscript i-s means ion-solvent potential energy and n-s means neutral halogen-solvent potential energy, $U_{p}^{\text {ion }}$ is the total polarization energy of the ionic cluster, and $U_{p}^{p}$ thl is the total polarization energy of the neutral cluster.

The experimental temperature, which is cluster-size dependent, is estimated roughly by using the evaporative en${\text { semble } \text { model }^{24} \text { following Lisy and co-workers. }}^{25}$ The inputs to this calculation are stepwise binding enthalpies, measured experimentally for the small clusters by Hiraoka et al., ${ }^{22}$ and the evaporation TOF between the cluster source and photo- electron spectrometer. For $\mathrm{I}^{-}\left(\mathrm{CH}_{3} \mathrm{CN}\right)_{n}$ clusters, the estimated upper bound for the temperature ranges from about $160 \mathrm{~K}$ for $n=4$ to about $70 \mathrm{~K}$ for $n=50$.

In order to explore as many configurations as possible within a limited computational effort, we perform several heating-cooling cycles during a MD run. The initial configurations are equilibrated at $200-300 \mathrm{~K}$ for a period of 501000 ps (depending on cluster size). Subsequently, clusters are cooled to the estimated experimental temperature, run for about $400 \mathrm{ps}$, and periodically heated to $200-300 \mathrm{~K}$ for brief periods of about $40 \mathrm{ps}$. Since both the configurations of the clusters and $E_{\text {stab }}$ are temperature dependent, data are collected only during the colder parts of the trajectories.

\section{RESULTS AND DISCUSSION}

\section{A. Experimental observations}

We have measured the PES of halogen anions solvated in $n$ acetonitrile molecules, where $n=1-33,1-40$, and $1-55$ for $\mathrm{Cl}^{-}, \mathrm{Br}^{-}$, and $\mathrm{I}^{-}$, respectively. The spectra of $\mathrm{I}^{-}, \mathrm{Br}^{-}$, and $\mathrm{Cl}^{-}$clustered with $\left(\mathrm{CH}_{3} \mathrm{CN}\right)_{n}$ are shown in Figs. 1, 2, and 3 .

In our analysis we postulate that the peaks of these spectra represent the average vertical binding energies of electrons in the clusters $\left[\mathrm{BE}_{\nu}(n)\right]$. We also assume that in these clusters the excess charge is practically localized on the anions. This hypothesis is supported by the recent experiments of Johnson and co-workers. ${ }^{26}$ We estimate the uncertainties in the vertical binding energies to be of the order of $15 \mathrm{meV}$ (for small clusters with $n=1-3$ ) up to $150 \mathrm{meV}$ [for the largest $\mathrm{X}^{-}\left(\mathrm{CH}_{3} \mathrm{CN}\right)_{n}$ clusters]. The difference between the vertical binding energy of a cluster and the electron affinity of the bare ion is defined as $E_{\text {stab }}(n)$ :

$E_{\text {stab }}(n)=\mathrm{BE}_{\nu}(n)-\mathrm{BE}_{\nu}(0)$.

$E_{\text {stab }}(n)$ is essentially the electrostatic stabilization of the solvated anion in its equilibrium configuration. In the vertical photodetachment process, the neutral halogen-solvent distances in the final state are identical to these of the anionsolvent, making the neutral atom-solvent interaction relatively weak. The simulations give neutral-solvent interaction energies which are up to $4 \%$ of the ion-solvent stabilization energy. Our experimental values of $E_{\text {stab }}(n)$ are listed in Table II together with the values of $E_{\text {stab }}(\mathrm{MD})$.

The scaled experimental $E_{\text {stab }}(n)$ values for the three halides are plotted in Fig. 4 as a function of cluster size. The 


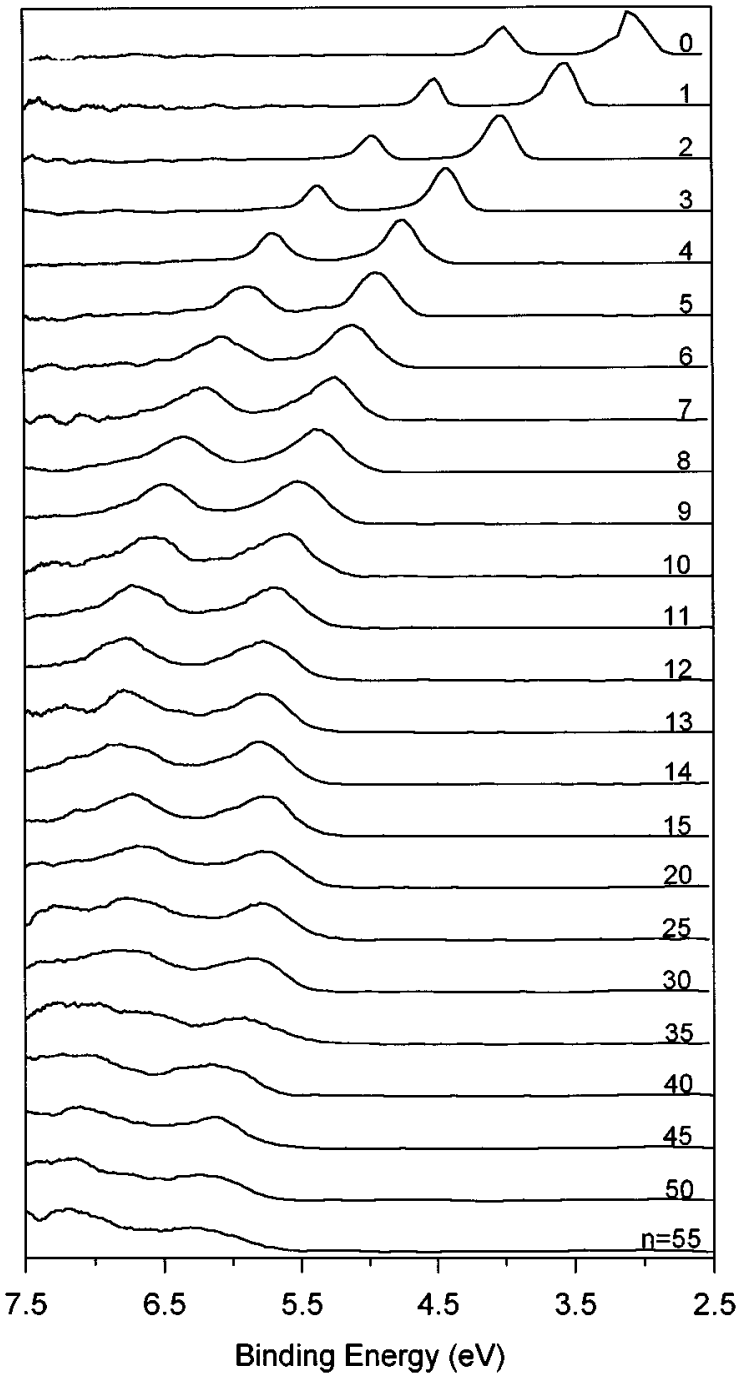

FIG. 1. Photoelectron spectra of $\mathrm{I}^{-}\left(\mathrm{CH}_{3} \mathrm{CN}\right)_{n}$ in the range of $n=1-55$, taken with $7.9 \mathrm{eV}$ photon energy.

scaling was done by dividing $E_{\text {stab }}(n)$ for each halide by $E_{\text {stab }}(1)$ of the same halide. In this way, a unified scale, in units of one acetonitrile-anion stabilization energy, is obtained. Note that iodide and bromide data fall on the same curve (within the experimental error), while the chloride stabilization energy steps are diminishing faster. The deviation of the chloride curve from the other two is mainly due to the abnormally high $E_{\text {stab }}(1)$ of the chloride. The PES stabilization energy of the complex of $\mathrm{Cl}^{-}$(solvent) ${ }_{1}$, both in water and acetonitrile, is inexplicably much higher than the measured binding enthalpy of the clusters. ${ }^{22}$ The binding enthalpy for such a small cluster should be close to the stabilization energy due to the absence of solvent-solvent interactions. ${ }^{27}$ Also note that in the case of the $\mathrm{Cl}^{-}\left(\mathrm{CH}_{3} \mathrm{CN}\right)_{1}$ cluster, we were unable to reproduce the PES stabilization energy in the MD simulations ( $0.24 \mathrm{eV}$ deviation), by using the same potential parameters which fitted well (within 0.05 $\mathrm{eV}$ ) the rest of the clusters. We suggest that in this cluster there is a very strong spectroscopic effect in the PES, resulting in intramolecular vibrational excitation in the neutral

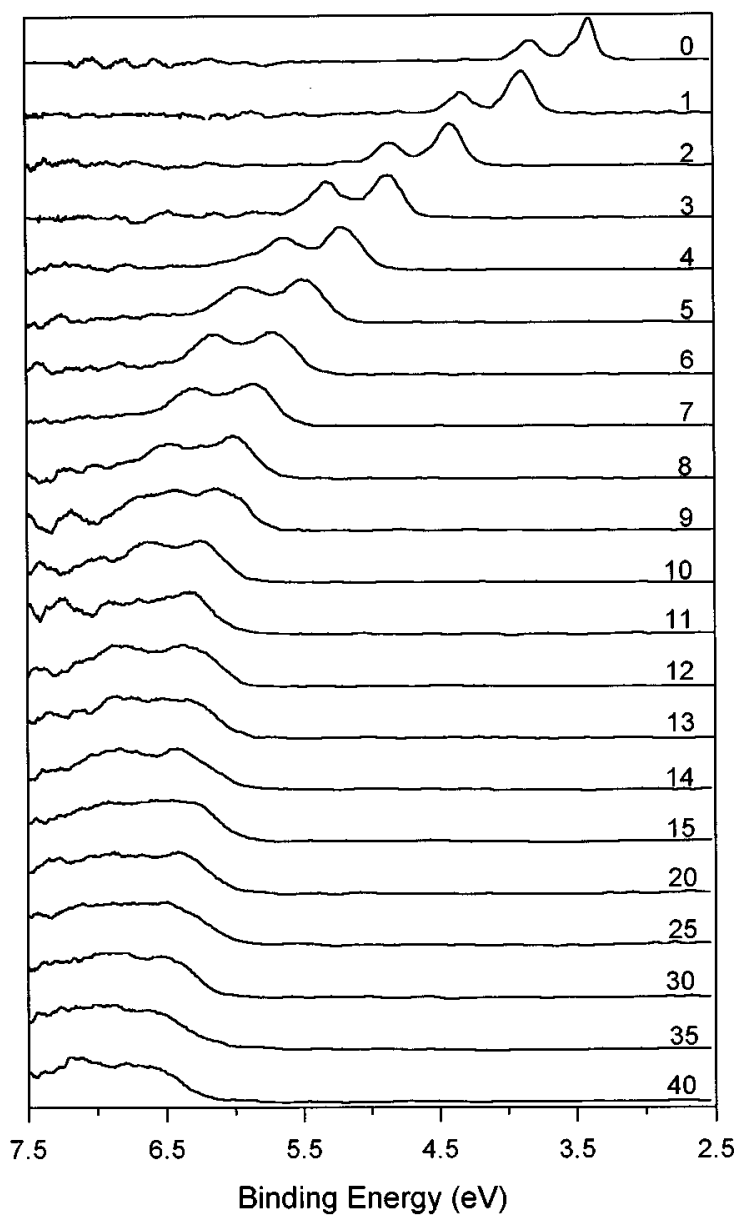

FIG. 2. Photoelectron spectra of $\mathrm{Br}^{-}\left(\mathrm{CH}_{3} \mathrm{CN}\right)_{n}$ in the range of $n=1-40$, taken with $7.9 \mathrm{eV}$ photon energy.

cluster. Such an effect would not be reproduced in our simulations which use rigid solvent molecules. Therefore, we tried to scale the chloride curve by dividing the $E_{\text {stab }}(n)$ values by half of the $E_{\text {stab }}(2)$. In this case, Fig. 4 reveals a good fit between the curves of the three halide-solvent clusters up to $n=9$.

A general trend in the scaled $E_{\text {stab }}(n)$ of all clusters is the gradual decrease in the differential stabilization energy, $\Delta E_{\text {stab }}(n)$, upon the stepwise addition of solvent molecules, $\Delta E_{\text {stab }}(n)=E_{\text {stab }}(n)-E_{\text {stab }}(n-1)$. This trend is well illustrated in Fig. 5. We attribute the decrease in differential stabilization to the influence of solvent-solvent interactions on the structure, which results in reduced ion-solvent interaction energy.

A common behavior to the three curves in Fig. 4 is the leveling of $E_{\text {stab }}(n)$ beyond $n=12$ for $\mathrm{I}^{-}, n=10-11$ for $\mathrm{Br}^{-}$, and $n=9-11$ for $\mathrm{Cl}^{-}$. This abrupt change in $E_{\text {stab }}(n)$ was not observed with other solvents $\left(\mathrm{H}_{2} \mathrm{O}, \mathrm{CO}_{2}, \mathrm{Ar}\right)$ in other PES experiments. We associate this phenomena with the completion of the first solvation layer around the halide. The absence of further stabilization of the anion with cluster size, reflects the switching in the relative strength of anionsolvent versus solvent-solvent interactions. In the first solvation layer, the relatively strong ion-solvent interactions, 


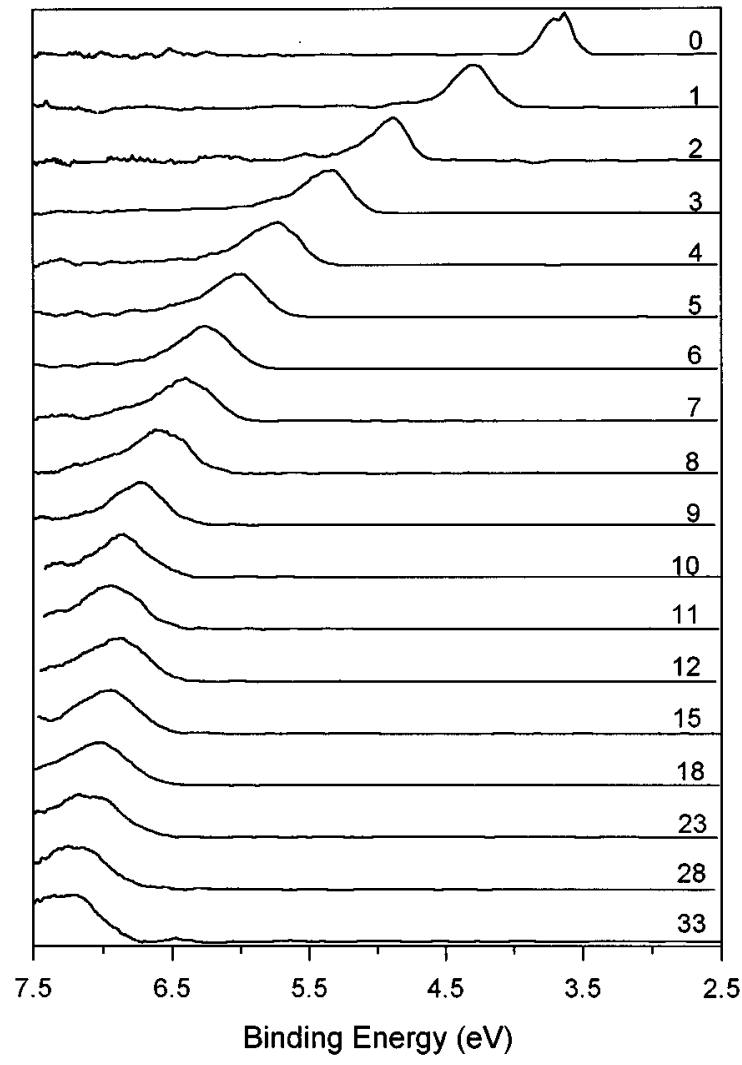

FIG. 3. Photoelectron spectra of $\mathrm{Cl}^{-}\left(\mathrm{CH}_{3} \mathrm{CN}\right)_{n}$ in the range of $n=1-33$, taken with $7.9 \mathrm{eV}$ photon energy.

which govern the cluster structure, result in efficient anion stabilization. In contrast, in the second layer, solvent-solvent interactions defeat the much weaker ion-solvent interactions, contributing no further stabilization to the anion.

At $n=9-20$, the chloride curve is somewhat lower in scaled stabilization energy than the other two. This is probably due to the smaller number of solvent molecules in the first layer around the smaller chloride.

Changes in the character of the clusters are reflected also in the mass spectra of the $\mathrm{X}^{-}\left(\mathrm{CH}_{3} \mathrm{CN}\right)_{n}$. There is a sharp drop in cluster abundance beyond $n=12,9$, and 9 for $\mathrm{I}^{-}$, $\mathrm{Br}^{-}$, and $\mathrm{Cl}^{-}$, respectively (see Fig. 6). Although the shape of cluster size distribution may be changed according to clustering conditions at the source, these sharp drops do not depend on source conditions. These sharp changes in abundance in the mass spectrum will be discussed in relation to our MD simulations.

\section{B. Simulation results}

The MD simulations provide a rationalization for the decrease in differential stabilization energy with increasing cluster size in the small clusters regime (Fig. 5).

Maximum stabilization of the halogen anion with one acetonitrile is fulfilled in a geometry in which the symmetry axis of acetonitrile is normal to the anion sphere with all three hydrogens equally distant from the anionic center. ${ }^{28}$ The clusters with $n=2-4$ maximize ion-solvent interaction by having the same ion-solvent configurations as in $n=1$ while minimizing repulsion between the ligands. The resulting structures are antiparallel, collinear for $n=2$, planartriangular structure for $n=3$, and roughly tetrahedral structure for $n=4$. In Fig. 7 we display snapshots from the simulations of a few small $\mathrm{I}^{-}\left(\mathrm{CH}_{3} \mathrm{CN}\right)_{n}$ clusters.

For larger clusters $(n>4)$, as the congestion of ligands around the anion increases, the acetonitrile ligands bend from the normal to the anion surface and the solvent-solvent interaction becomes attractive, with the penalty of a reduced differential $E_{\text {stab }}$. For instance, the solvent-solvent Coulomb energy for $\mathrm{I}^{-}\left(\mathrm{CH}_{3} \mathrm{CN}\right)_{4}$ is $+160 \mathrm{meV}$, while for $\mathrm{I}^{-}\left(\mathrm{CH}_{3} \mathrm{CN}\right)_{6}$ it is already $-80 \mathrm{meV}$. This change can be noted in the average ion-hydrogen radial distribution function for the $n=4,5$ clusters in Fig. 8. The behavior is common to the three halides: At $n=4$ all three hydrogen atoms of each acetonitrile molecule are nearly equivalent. At $n=5$ two hydrogen atoms become closer to the anion while the third is further away, interacting with the nearest-neighbor nitrogen atom, and two separate peaks appear in the ion-hydrogen distance distribution function. In larger clusters, some of the molecules have one hydrogen in contact with the anion and the other two pointing toward the second solvation layer.

The $\mathrm{I}^{-}\left(\mathrm{CH}_{3} \mathrm{CN}\right)_{6}$ and $\mathrm{Br}^{-}\left(\mathrm{CH}_{3} \mathrm{CN}\right)_{6}$ clusters possess a common structure: The acetonitrile molecules form a planar ring around the ion, having head to tail interactions between the molecules, and one or two hydrogen atoms in contact with the ion, optimizing both solvent-solvent and ionsolvent interactions. $\mathrm{Cl}^{-}\left(\mathrm{CH}_{3} \mathrm{CN}\right)_{6}$ is too crowded to form a ring and the molecules move out of the plane of the ring.

Extreme care has to be taken in the simulations of larger clusters. Depending on the way the trajectories are run, different structures may result. The way the clusters are annealed in order to obtain the "experimental" structure is crucial to both structure and $E_{\text {stab }}$. One important example in this work is the $\mathrm{I}^{-}\left(\mathrm{CH}_{3} \mathrm{CN}\right)_{12}$ cluster. On running a long trajectory (a few ns) at $110-120 \mathrm{~K}$, which is the estimated upper bound to the experimental temperature, with periodic heating to $200 \mathrm{~K}$, the cluster would have one solvent molecule at the second solvation shell, on the average. No special order appears in the first solvation layer and the experimental stabilization energy is reproduced within $40 \mathrm{meV}$. If one uses very short (2-3 ps) heating periods to $300 \mathrm{~K}$, which would cause dissociation on a longer time scale, and quenches them to a relatively low temperature $(70 \mathrm{~K})$, then a much more stable structure appears. This "crystalline" structure is about $200 \mathrm{meV}$ lower in total energy than the former "amorphous" structure, while having $E_{\text {stab }}(12)$ which is smaller by about $200 \mathrm{meV}$ than the experimental one. It has all the 12 molecules in the first layer, forming distorted cyclic trimers which are arranged in a symmetric shell, having a $T$ point group symmetry (Fig. 9). This stable structure can survive heating up to $150 \mathrm{~K}$ for several nanoseconds.

Another example is $n=11$. Regular trajectories with periodic heating produce equilibrium configurations having one solvent molecule in the second layer of $\mathrm{I}^{-}\left(\mathrm{CH}_{3} \mathrm{CN}\right)_{11}$. When an initial configuration of this cluster is produced by remov- 
TABLE II. Experimental and simulated $E_{\text {stab }}$ values of the $\mathrm{X}^{-}\left(\mathrm{CH}_{3} \mathrm{CN}\right)_{n}$ clusters. The temperatures at which the simulated $E_{\text {stab }}$ values were collected, and the number of solvent molecules in the first solvation layer are indicated. We estimate the errors in determining experimental $E_{\text {stab }}$ to be of the order of 15 meV for $n=1$ up to $150 \mathrm{meV}$ for $n=55$. Uncertainties in simulated $E_{\text {stab }}$ are of the same order of magnitude. The numbers in parenthesis refer to stable structures which are hardly accessible through MD trajectories.

\begin{tabular}{|c|c|c|c|c|c|c|c|c|c|c|c|c|}
\hline \multirow[b]{2}{*}{$n$} & \multicolumn{4}{|c|}{$\mathrm{Cl}^{-}$} & \multicolumn{4}{|c|}{$\mathrm{Br}^{-}$} & \multicolumn{4}{|c|}{$\mathrm{I}^{-}$} \\
\hline & $\begin{array}{l}E_{\text {stab }} \\
\text { exp. }\end{array}$ & $\begin{array}{l}E_{\text {stab }} \\
\text { sim. }\end{array}$ & $\begin{array}{c}T_{\text {sim }} \\
(\mathrm{K})\end{array}$ & $\begin{array}{c}\text { Solv. } \\
\text { no. }\end{array}$ & $\begin{array}{l}E_{\text {stab }} \\
\text { exp. }\end{array}$ & $\begin{array}{l}E_{\text {stab }} \\
\text { sim. }\end{array}$ & $\begin{array}{c}T_{\text {sim }} \\
(\mathrm{K})\end{array}$ & $\begin{array}{c}\text { Solv. } \\
\text { no. }\end{array}$ & $\begin{array}{l}E_{\text {stab }} \\
\text { exp. }\end{array}$ & $\begin{array}{l}E_{\text {stab }} \\
\text { sim. }\end{array}$ & $\begin{array}{l}T_{\text {sim }} \\
(\mathrm{K})\end{array}$ & $\begin{array}{c}\text { Solv. } \\
\text { no. }\end{array}$ \\
\hline 1 & 0.73 & 0.59 & 180 & 1 & 0.54 & 0.53 & 170 & 1 & 0.48 & 0.49 & 170 & 1 \\
\hline 2 & 1.25 & 1.18 & 170 & 2 & 1.04 & 1.04 & 170 & 2 & 0.96 & 0.95 & 170 & 2 \\
\hline 3 & 1.77 & 1.71 & 170 & 3 & 1.49 & 1.50 & 170 & 3 & 1.38 & 1.40 & 160 & 3 \\
\hline 4 & 2.16 & 2.14 & 170 & 4 & 1.86 & 1.86 & 160 & 4 & 1.70 & 1.75 & 160 & 4 \\
\hline 5 & 2.41 & 2.38 & 160 & 5 & 2.11 & 2.09 & 160 & 5 & 1.89 & 1.88 & 150 & 5 \\
\hline 6 & 2.66 & 2.68 & 150 & 6 & 2.35 & 2.34 & 150 & 6 & 2.05 & 2.06 & 140 & 6 \\
\hline 7 & 2.83 & 2.81 & 150 & 7 & 2.48 & 2.43 & 150 & 7 & 2.21 & 2.22 & 140 & 7 \\
\hline 8 & 2.99 & 2.82 & 140 & $7-8$ & 2.64 & 2.60 & 140 & 8 & 2.34 & 2.25 & 130 & 8 \\
\hline 9 & 3.14 & 3.05 & 130 & 9 & 2.76 & 2.61 & 130 & 9 & 2.45 & 2.33 & 120 & 9 \\
\hline 10 & 3.23 & 3.22 & 130 & 10 & 2.91 & 2.82 & 130 & 9 & 2.56 & 2.44 & 120 & 9 \\
\hline 11 & 3.31 & 3.32 & 130 & 10 & 2.96 & 2.92 & 130 & 10 & 2.65 & $\begin{array}{c}2.62 \\
(2.52)\end{array}$ & 120 & $\begin{array}{c}10 \\
(11)\end{array}$ \\
\hline 12 & 3.29 & 3.37 & 120 & 11 & 3.01 & 3.00 & 120 & 11 & 2.74 & $\begin{array}{c}2.69 \\
(2.53)\end{array}$ & 115 & $\begin{array}{c}11 \\
(12)\end{array}$ \\
\hline 13 & 3.33 & 3.35 & 120 & 12 & 3.03 & & & & 2.74 & 2.74 & 110 & 13 \\
\hline 14 & 3.31 & & & & 3.06 & & & & 2.70 & & & \\
\hline 15 & 3.31 & 3.43 & 115 & 11 & 3.06 & 3.09 & 115 & 12 & 2.70 & 2.82 & 110 & 13 \\
\hline 16 & & & & & & & & & 2.70 & & & \\
\hline 17 & & & & & 3.08 & & & & & & & \\
\hline 18 & 3.35 & 3.45 & 100 & $11-12$ & & & & & & & & \\
\hline 20 & & & & & 3.06 & & & & 2.69 & 2.81 & 100 & 13 \\
\hline 23 & 3.47 & 3.45 & 100 & 11 & & & & & & & & \\
\hline 25 & & & & & 3.15 & 3.16 & 100 & 12 & 2.72 & 2.84 & 90 & $12-13$ \\
\hline 28 & 3.56 & & & & & & & & & & & \\
\hline 30 & & & & & 3.19 & & & & 2.81 & & & \\
\hline 33 & 3.62 & & & & & & & & & & & \\
\hline 35 & & & & & 3.24 & & & & 2.89 & & & \\
\hline 40 & & & & & 3.28 & & & & 3.09 & & & \\
\hline 45 & & & & & & & & & 3.07 & & & \\
\hline 50 & & & & & & & & & 3.18 & & & \\
\hline 55 & & & & & & & & & 3.21 & & & \\
\hline
\end{tabular}

ing one molecule from the more stable form of the $\mathrm{I}^{-}\left(\mathrm{CH}_{3} \mathrm{CN}\right)_{12}$, or by quenching from very high to low temperature $(70 \mathrm{~K})$, a more stable form of the cluster is obtained. This isomer has all the 11 solvent molecules in the first solvation layer. It is lower by about $100 \mathrm{meV}$ in total energy than the one obtained in the regular runs, and again, while the stabilization energy obtained from the regular run fits the experimental $E_{\text {stab }}(11)$ within $30 \mathrm{meV}$, this quantity is smaller in the more stable isomer by $120 \mathrm{meV}$.

We have also tried to find a more stable configuration of the $\mathrm{I}^{-}\left(\mathrm{CH}_{3} \mathrm{CN}\right)_{13}$ cluster by taking the most stable $n=12$ cluster, adding a molecule to the second solvation layer, and running for a few nanoseconds at $110 \mathrm{~K}$. The additional molecule moved into the first layer, producing the same disordered structure that was achieved previously in the regular heating-cooling cycles. Note, that on going from the stable form of the $\mathrm{I}^{-}\left(\mathrm{CH}_{3} \mathrm{CN}\right)_{12}$ cluster to the $\mathrm{I}^{-}\left(\mathrm{CH}_{3} \mathrm{CN}\right)_{13}$ cluster, the total energy of the cluster is lowered by $320 \mathrm{meV}$, while on going from $n=11$ to 12 it lowers by $450 \mathrm{meV}$. The changes in the solvent-solvent interaction energy are more illuminating: It becomes more negative by about $400 \mathrm{meV}$ between the most stable forms of $n=11$ and 12 while no change is observed between $n=12$ and 13 .

Looking for a similar behavior in the bromide and chloride clusters, we found that both $\mathrm{Br}^{-}\left(\mathrm{CH}_{3} \mathrm{CN}\right)_{9}$ and $\mathrm{Cl}^{-}\left(\mathrm{CH}_{3} \mathrm{CN}\right)_{9}$ clusters, as in the case of the $\mathrm{I}^{-}\left(\mathrm{CH}_{3} \mathrm{CN}\right)_{12}$, consist of distorted cyclic trimers of acetonitrile. In bromide and chloride with $n=9$ the acetonitrile cage is not complete and the symmetry is lower. A snapshot of the $\mathrm{Cl}^{-}\left(\mathrm{CH}_{3} \mathrm{CN}\right)_{9}$ cluster is shown in Fig. 10. In this case, the changes in total cluster energy between $n=8$ and 9 and between $n=9$ and 10 are almost constant, while the changes in the solvent-solvent energy are those which contain valuable information. Both in bromide and chloride, on going from $n=7$ to 8 and from $n=8$ to 9 , the solvent-solvent energy becomes 300-400 $\mathrm{meV}$ more negative, while on going from $n=9$ to 10 , it is reduced by less than $100 \mathrm{meV}$. We believe that the intensity drops in the mass spectrum of the $\mathrm{X}^{-}\left(\mathrm{CH}_{3} \mathrm{CN}\right)_{n}$ clusters are related to these observations. The ordered cyclic trimer structures of the solvent in the $\mathrm{Cl}^{-}, \mathrm{Br}^{-}, \mathrm{I}^{-}$clusters with $n=9,9$, and 12, respectively, have relatively low total energy and very low solvent-solvent energy which makes the enthalpy 


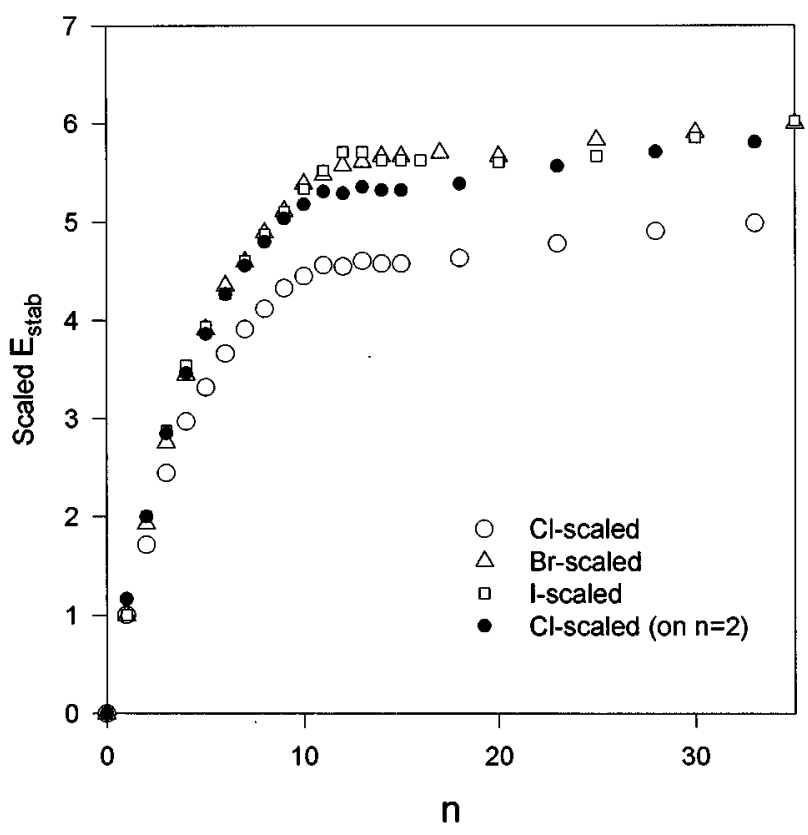

FIG. 4. Scaled stabilization energies as a function of cluster size for $\mathrm{X}^{-}\left(\mathrm{CH}_{3} \mathrm{CN}\right)_{n}$ clusters where $\mathrm{X}=\mathrm{Cl}, \mathrm{Br}$, and $\mathrm{I}$. The scaling was done by dividing $E_{\text {stab }}(n)$ by $E_{\text {stab }}(1)$ for each halide. For chloride, a scaling by division by $E_{\text {stab }}(2) / 2$ is also presented (full circles) - see the text for an explanation.

of association of these clusters more negative with respect to the other, amorphous, clusters. Consequently, these clusters are less susceptible to evaporation of acetonitrile molecules, leading to a "magic number" in the mass spectrum. As pointed out recently by Sremaniak, Perera, and Berkewitz ${ }^{29}$ structures and phase transitions in such systems are often

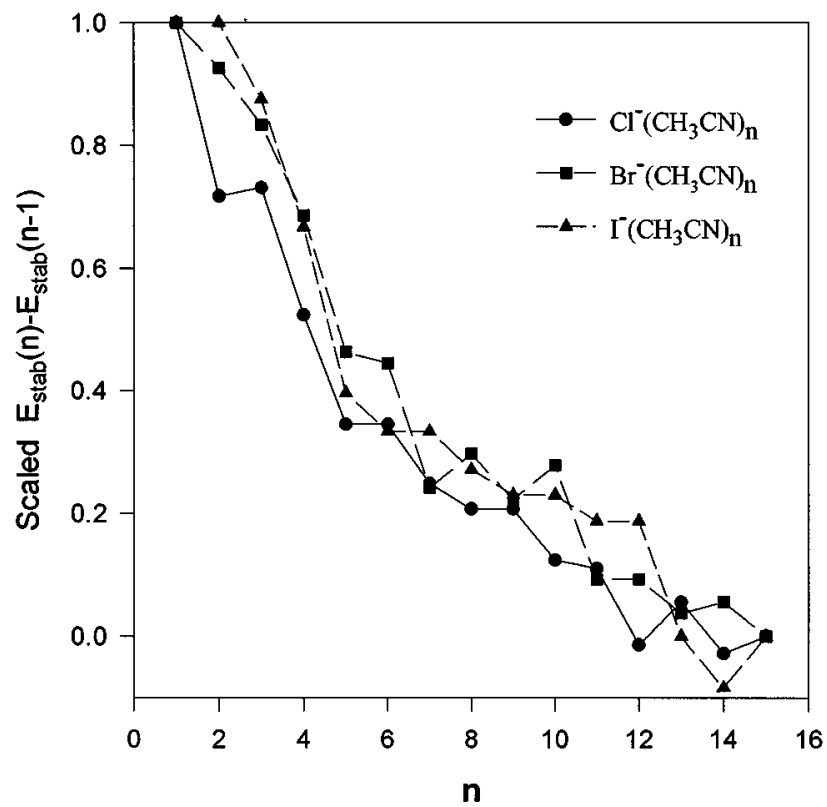

FIG. 5. Scaled differential stabilization energies as a function of cluster size for $\mathrm{X}^{-}\left(\mathrm{CH}_{3} \mathrm{CN}\right)_{n}$ clusters where $\mathrm{X}=\mathrm{Cl}, \mathrm{Br}$, and $\mathrm{I}$. The scaling was done by dividing $\left[E_{\text {stab }}(n)-E_{\text {stab }}(n-1)\right]$ by $E_{\text {stab }}(1)$ for each halide.

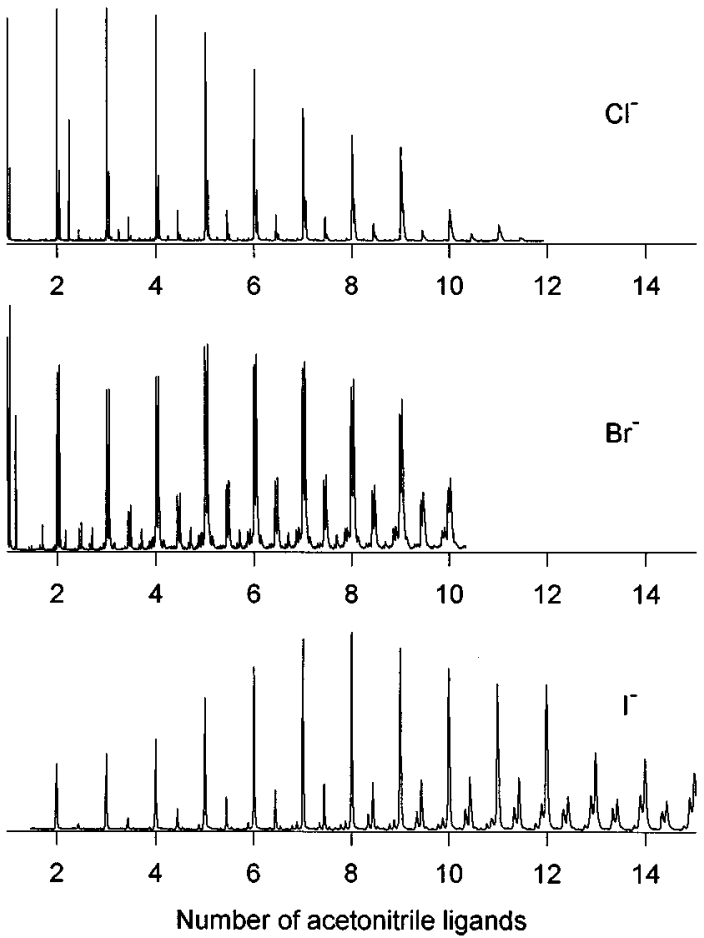

FIG. 6. Typical mass spectra of $\mathrm{X}^{-}\left(\mathrm{CH}_{3} \mathrm{CN}\right)_{n}$ cluster series where $\mathrm{X}=\mathrm{Cl}$, $\mathrm{Br}$, and $\mathrm{I}$. Note the sharp drops in intensity beyond $n=9,9$, and 12 for $\mathrm{Cl}^{-}$, $\mathrm{Br}^{-}$, and $\mathrm{I}^{-}$, respectively. These drops are unique throughout the series and do not disappear upon changes in cluster size distribution.

governed by solvent-solvent interactions, while the properties of the ion play a secondary part in such phenomena.

In discussing the reliability of our simulations, one must bear in mind that these structural properties are not extremely sensitive to the exact form of the potential used in the simulation. Increasing the atomic charge distribution by $5 \%$, or taking in or out the polarization term, did not change the structures significantly. $E_{\text {stab }}(n)$ values, however, were very sensitive to the potential parameters that were used and did not easily fit the experimental ones. Furthermore, $E_{\text {stab }}(n)$ was found to be very sensitive to the exact configuration of the molecules in the first solvation layer, which may change upon changing the temperature of a cluster or trapping in different local minima. Further discussion of phase transitions and temperature effects in MD simulations of halide-water clusters can be found in Ref. 29.

Beyond the first solvation layer $(n>12)$, the additional acetonitrile ligands form disordered structures, maximizing the interaction with the molecules in the first layer and among themselves. These molecules usually form nonlinear head-to-tail configurations and less frequently-antiparallel pairs. The dipole moments of the molecules in the second layer are roughly perpendicular to the ion-molecule vector, adding no stabilization to the anion. They may rather distort the structure of the first solvation layer, yielding negative stabilization of the anion. This is a fine example of "structure breaking" geometries which is traditionally attributed to second solvation layers in the bulk. 

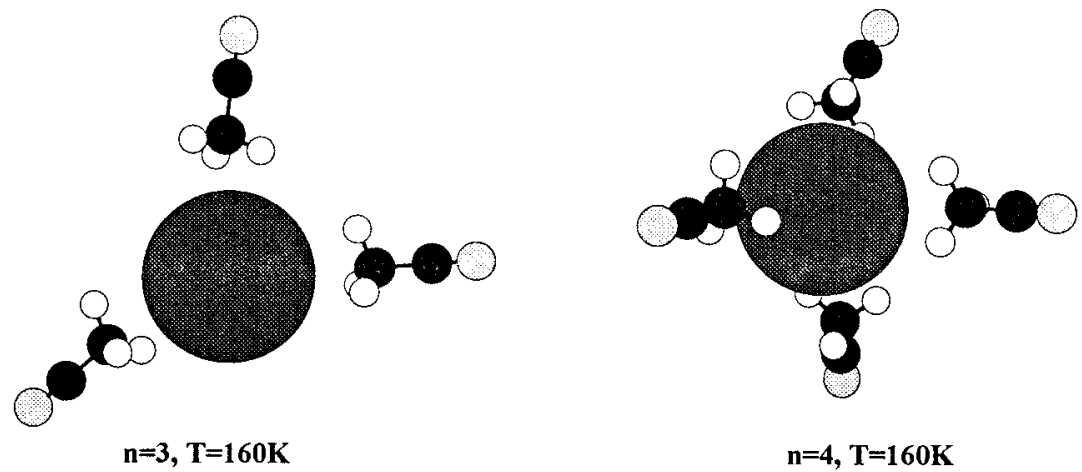

$\mathrm{n}=4, \mathrm{~T}=160 \mathrm{~K}$
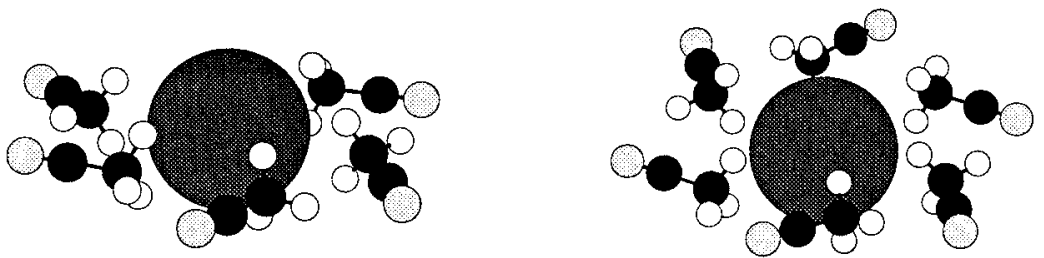

$n=5, T=150 K$

$n=6, T=140 K$

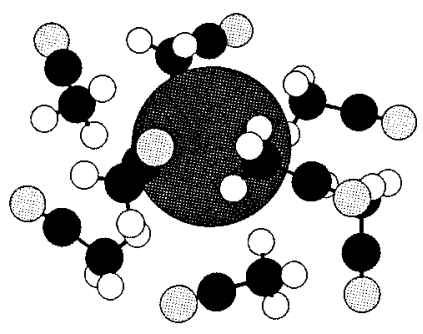

$\mathrm{n}=\mathbf{8}, \mathrm{T}=130 \mathrm{~K}$

FIG. 7. Structures of small $\mathrm{I}^{-}\left(\mathrm{CH}_{3} \mathrm{CN}\right)_{n}$ clusters, calculated in the MD simulations. The estimated experimental temperatures, at which the data were collected, are indicated. van der Waals radii of atoms in acetonitrile are scaled down by 0.3 for visual clarity.

The largest clusters we have simulated, $\mathrm{I}^{-}$and $\mathrm{Br}^{-}$with 25 solvent molecules, and $\mathrm{Cl}^{-}$with 23 molecules, had 12 13, 12, and 11 solvent molecules, respectively, in the first solvation layer at $90-110 \mathrm{~K}$, having the outer solvent molecules concentrated over a limited solid angle around the

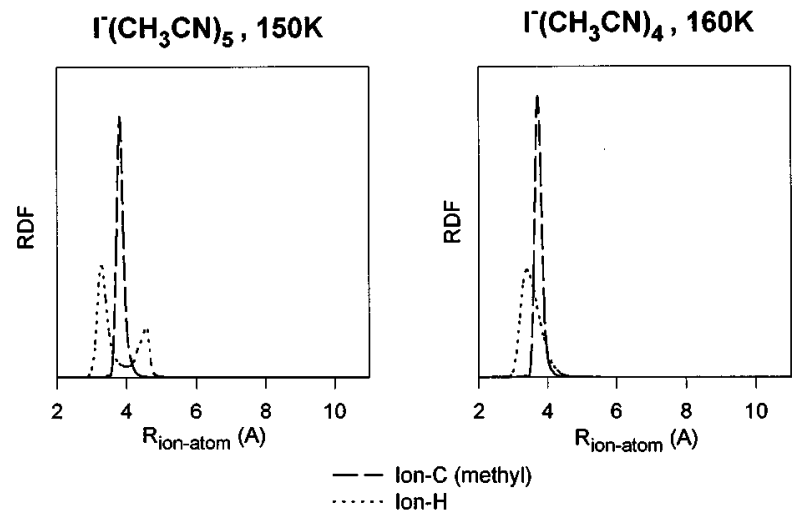

FIG. 8. $\mathrm{I}^{-}-\mathrm{H}$ and $\mathrm{I}^{-}-\mathrm{C}$ (methyl) radial distribution functions, accumulated over $0.5 \mathrm{~ns}$ runs of $\mathrm{I}^{-}\left(\mathrm{CH}_{3} \mathrm{CN}\right)_{n}$ clusters with $n=4,5$ at $T=160-150 \mathrm{~K}$. anion. A typical snapshot of the $\mathrm{I}^{-}\left(\mathrm{CH}_{3} \mathrm{CN}\right)_{25}$ cluster is presented in Fig. 11. This trend may probably continue in larger clusters: The second solvation layer might not be completed, building an acetonitrile drop which is attached to the first solvation layer. The anion-solvent stabilization added by the molecules beyond the first solvation layer is small relative to solvent-solvent interaction energy. Therefore, the solvent would rather form a drop and not fill the second layer. The small gradual increase in experimental $E_{\text {stab }}(n)$ beyond $n=20-25$ can be attributed to the electrostatic polarization of the solvent drop beyond the first solvent shell (Born energy).

The evolution of $E_{\text {stab }}(n)$ in halide-acetonitrile cluster is distinctly different from that of halide-water clusters (Fig. 12). Note, that the water clusters do not show any leveling of $E_{\text {stab }}(n)$ (only a change in slope at $n=6$ ). Already at small sizes, the halide-water clusters have structures that resemble pure water structures, ${ }^{5,29}$ while the halide-acetonitrile clusters probably need tens of solvent molecules to start building solvent-solvent structures which resemble the acetonitrile bulk structure. In the $\mathrm{X}^{-}\left(\mathrm{CH}_{3} \mathrm{CN}\right)_{n}$ clusters, the first solvation layer of the clusters is full (for $n>12$ ), with 11-13 


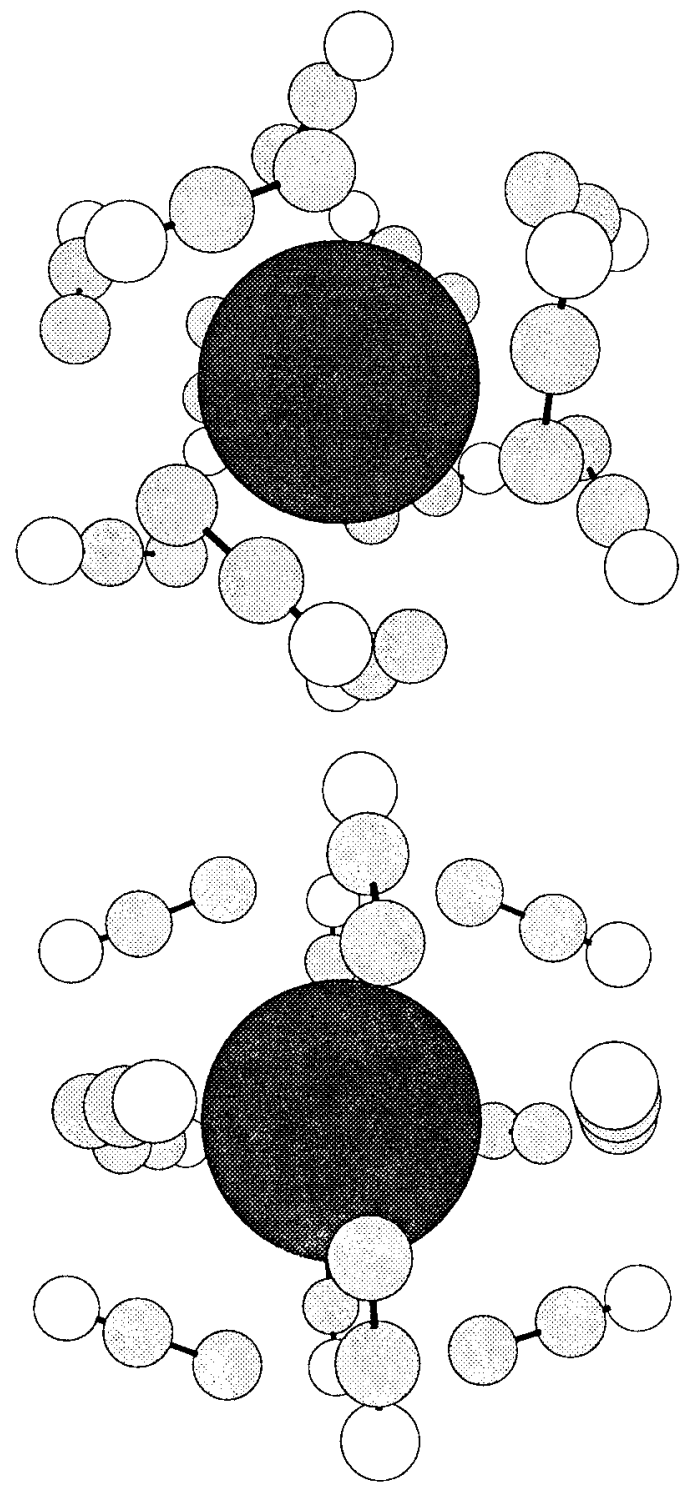

FIG. 9. The structure of the highly stable form of the $\mathrm{I}^{-}\left(\mathrm{CH}_{3} \mathrm{CN}\right)_{12}$ cluster. Hydrogens are not shown in the picture for visual clarity. Nitrogen is represented by the clear circles. All the acetonitrile molecules are combined in cyclic trimers. Two viewing angles are presented: On the top, one of the four $C_{3}$ axes of the $T$ point group is perpendicular to the plane of the paper. On the bottom, one of the three $C_{2}$ axes.

solvent molecules in this layer, while in halide-water clusters, the first solvation layer is only partially filled, with a much smaller solvation number.

The marked difference in the structure of the solvent in the first solvation layer, between water and acetonitrile, fades away on moving beyond the first layer. There, as in any polar solvent, the solvent-solvent interaction becomes larger than ion-solvent interaction and the system would maximize solvent-solvent interactions to form a densely packed drop of the solvent, rather than a spherical symmetric solvation shell around the ion. Thus, large ion-acetonitrile clusters will have a solvent drop attached to a full first solvation layer, while the water clusters may have the anion exposed at the surface of the drop. At large enough clusters and high
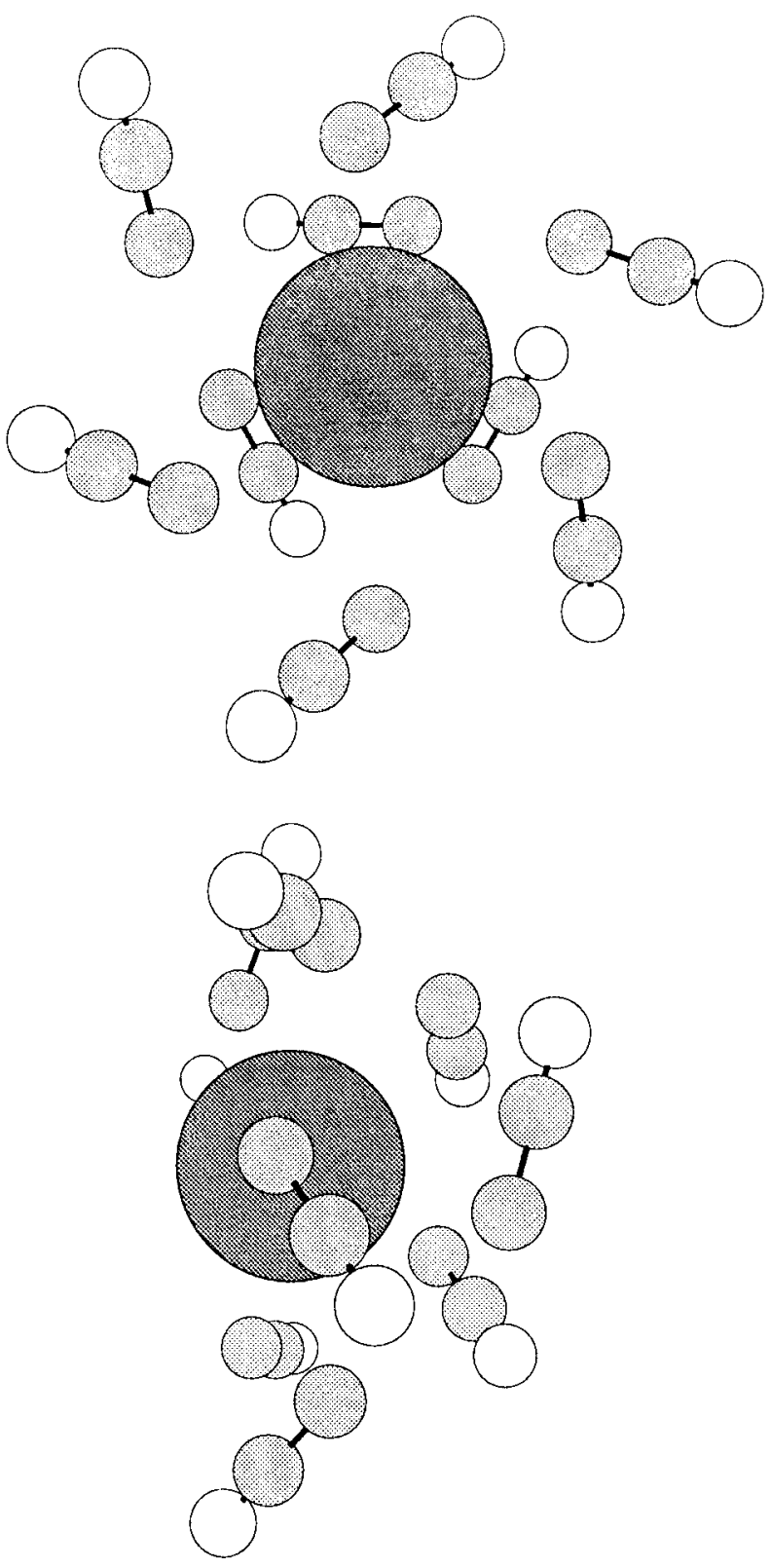

FIG. 10. The structure of the $\mathrm{Cl}^{-}\left(\mathrm{CH}_{3} \mathrm{CN}\right)_{9}$ cluster. As the $\mathrm{I}^{-}\left(\mathrm{CH}_{3} \mathrm{CN}\right)_{12}$ cluster, it is built out of cyclic acetonitrile trimers, but this time they do not form a full solvation shell. Hydrogens are not shown in the picture for visual clarity. Nitrogen is represented by the clear circles.

enough temperatures, the entropy and long range polarization energy may finally drive the anion toward the center of the cluster.

\section{CONCLUSIONS}

We have demonstrated the sensitivity of PES to the microscopic details of ion solvation. The pure ion-solvent electrostatic interaction, extracted from the PES experiment, shows significant differences between water and acetonitrile, reflecting differences in cluster solvation structures.

Classical molecular dynamics simulations, if used carefully, are indispensable in the analysis of results, in spite of 


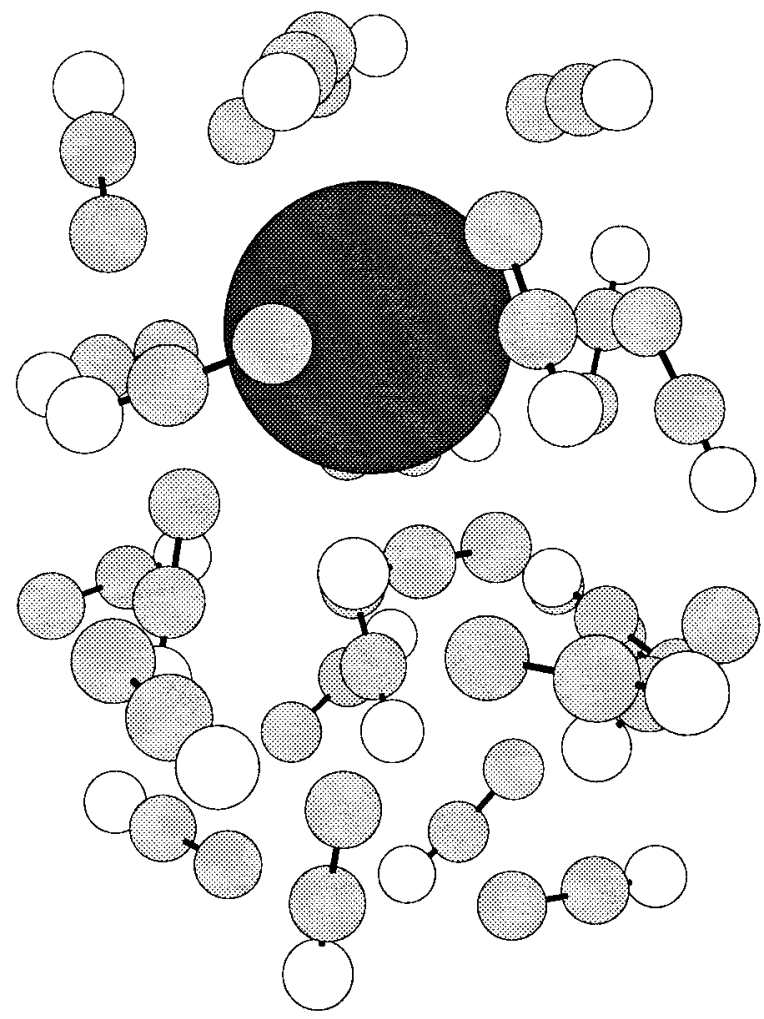

FIG. 11. A typical snapshot of the $\mathrm{I}^{-}\left(\mathrm{CH}_{3} \mathrm{CN}\right)_{25}$ cluster at $T=90 \mathrm{~K}$. Note that the molecules outside the first solvation layer are concentrated to form a drop that is attached to the first layer. Hydrogens are not shown in the picture for visual clarity. Nitrogen is represented by the clear circles.

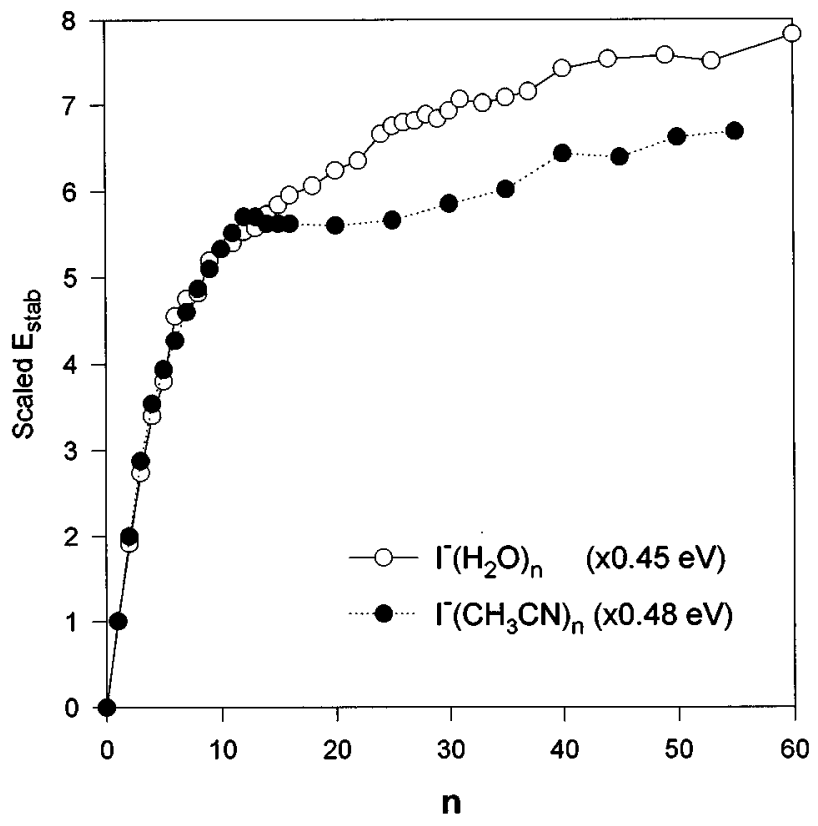

FIG. 12. Scaled stabilization energies of $\mathrm{I}^{-}\left(\mathrm{CH}_{3} \mathrm{CN}\right)_{n}$ and $\mathrm{I}^{-}\left(\mathrm{H}_{2} \mathrm{O}\right)_{n}$ clusters as a function of cluster size. For each solvent, the $E_{\text {stab }}(n)$ values are normalized to $E_{\text {stab }}(1)$. the rough potential functions employed. By finding an atomic charge distribution that produces better agreement between the simulation results and the experiments, we might have improved the potentials used so far in acetonitrile simulations. The anion-acetonitrile $E_{\text {stab }}$ calculations pose a more stringent test on the atomic charge distribution, in comparison with the pure acetonitrile simulations.

As revealed to us in the case of $\mathrm{I}^{-}\left(\mathrm{CH}_{3} \mathrm{CN}\right)_{12}$, finding the global minima of multidimensional systems such as ionacetonitrile clusters with tens of solvent molecules, using $\mathrm{MD}$, is a difficult, and sometimes unreliable task. It is possible, that the exploration of phase space toward the global minimum of such a complex system may be done more efficiently using other methods, employing random configuration changes in the search scheme. MD may be successfully applied afterwards, using the global minima as a starting point, to study dynamics, temperature effects, and structures of complex systems.

\section{ACKNOWLEDGMENTS}

The research was supported by the James Franck German-Israeli Binational Program in Laser Matter Interaction, and by the US-Israel Binational Science Foundation. G.M. is a Clore Scholar. The help of I. Schek in running the simulations is highly appreciated.

${ }^{1}$ S. T. Arnold, J. G. Eaton, D. Patel-Misra, H. W. Sarkas, and K. H. Bowen, in Ion and Cluster Ion Spectroscopy and Structure, edited by J. P. Maier (Elsevier, Amsterdam, 1989), p. 417.

${ }^{2}$ S. T. Arnold, J. H. Hendricks, and K. H. Bowen, J. Chem. Phys. 102, 39 (1995).

${ }^{3}$ D. W. Arnold, S. E. Bradforth, E. H. Kim, and D. M. Neumark, J. Chem. Phys. 102, 3510 (1995).

${ }^{4}$ M. J. Deluca, C. C. Han, and M. A. Johnson, J. Chem. Phys. 93, 268 (1990).

${ }^{5}$ G. Markovich, S. Pollack, R. Giniger, and O. Cheshnovsky, J. Chem. Phys. 101, 9344 (1994).

${ }^{6}$ P. Delahay, Acc. Chem. Res. 15, 40 (1982).

${ }^{7}$ R. E. Ballard, J. Jones, D. Read, A. Inchley, and M. Cranmer, Chem. Phys. Lett. 134, 177 (1987).

${ }^{8}$ M. Faubel, B. Steiner, and J. P. Toennies, Nature (submitted).

${ }^{9}$ Note, however, that the induced dipole solvent-solvent interactions, caused by the anion, do not exist in the neutral photodetachment product.

${ }^{10}$ L. Perera and M. L. Berkowitz, J. Chem. Phys. 95, 1954 (1991); L. S. Sremaniak, L. Perera, and M. L. Berkowitz, Chem. Phys. Lett. 218, 377 (1994).

${ }^{11}$ See also L. X. Dang and B. C. Garrett, J. Chem. Phys. 99, 2972 (1993).

${ }^{12}$ L. Perera and F. G. Amar, J. Chem. Phys. 93, 4884 (1990).

${ }^{13}$ F. G. Amar, S. Goyal, D. J. Levandier, L. Perera, and G. Scoles, in Clusters of Atoms and Molecules II, edited by H. Haberland (Springer, Heidelberg, 1994), p. 19.

${ }^{14}$ D. M. Neumark (private communication).

${ }^{15}$ H. J. Bohm, I. R. McDonald, and P. A. Madden, Mol. Phys. 49, 347 (1983).

${ }^{16}$ P. Kruit and F. H. Read, J. Phys. E 16, 313 (1983); O. Chesnovsky, S. H. Yang, C. L. Pettiette, M. J. Craycraft, and R. E. Smalley, Rev. Sci. Instrum. 58, 2131 (1987).

${ }^{17}$ H. Hotop and W. C. Lineberger, J. Phys. Chem. Ref. Data 4, 539 (1975).

${ }^{18}$ G. Del Mistro and A. J. Stace, J. Chem. Phys. 99, 4656 (1993); A. J. Stace and G. Del Mistro, ibid. 102, 5900 (1995).

${ }^{19}$ U. Buck and I. Ettischer, Faraday Discuss. Chem. Soc. 97, 215 (1994).

${ }^{20}$ C. Desfrancois, H. Abdoul-Carime, N. Khglifa, J. P. Schermann, V. Brenner, and P. Millie, J. Chem. Phys. 102, 4952 (1995).

${ }^{21}$ A. F. Jayaraj and S. Singh, J. Mol. Struct. 327, 107 (1994). 
${ }^{22}$ K. Hiraoka, S. Mizuse, and S. Yamabe, J. Phys. Chem. 92, 3943 (1988).

${ }^{23}$ J. Caldwell, L. X. Dang, and P. A. Kollman, J. Am. Chem. Soc. 112, 9144 (1990).

${ }^{24}$ C. E. Klotz, J. Phys. Chem. 92, 5864 (1988); C. E. Klotz, Z. Phys. D 5, 83 (1987).

${ }^{25}$ J. A. Draves, Z. Luthey-Schulten, W. L. Liu, and J. M. Lisy, J. Chem. Phys. 93, 4589 (1990).
${ }^{26}$ C. E. H. Dessent, J. Kim, and M. A. Johnson, J. Phys. Chem. 100, 12 (1996).

${ }^{27}$ G. Markovich, R. Giniger, M. Levin, and O. Cheshnovsky, Z. Phys. D 20, 69 (1991).

${ }^{28}$ This result is consistent with the $a b$ initio calculation of Ref. 21 .

${ }^{29}$ L. S. Sremaniak, L. Perera, and M. L. Berkowitz, J. Phys. Chem. 100, 1350 (1996) 